\title{
Conhecimento dos Profissionais de Enfermagem sobre Segurança do Paciente Oncológico em Quimioterapia
}

http://dx.doi.org/10.32635/2176-9745.RBC.2019v65n1.274

\author{
Knowledge of Nursing Professional on the Safety of the Oncological Patient in Chemoterapy \\ Conocimiento de los Profesionales de Enfermería sobre Seguridad del Paciente Oncológico en Quimioterapia
}

\begin{abstract}
Aline Gonçalves da Costa'; Marta Solange Camarinha Ramos Costa ${ }^{2}$; Elisângela da Silva Ferreira ${ }^{3}$; Priscila Cristina de Sousa ${ }^{4}$; Marília Monteiro dos Santos ${ }^{5}$; Danielle Etienne de Oliveira Bezerra Lima ${ }^{6}$; Aline Maria Pereira Cruz Ramos ${ }^{7}$
\end{abstract}

Resumo

Introduçáo: A segurança do paciente vem adquirindo grande importância em níveis nacional e internacional, especialmente na oncologia, na qual há uma preocupação crescente com os erros/eventos adversos relacionados às drogas quimioterápicas antineoplásicas, tendo como principal premissa a qualidade do cuidado em saúde e a assistência segura. Objetivo: Avaliar o conhecimento dos profissionais de enfermagem quanto à segurança do paciente oncológico em tratamento quimioterápico. Método: Estudo qualitativo, realizado no ambulatório de uma Unidade de Assistência de Alta Complexidade em Oncologia, localizado no Estado do Pará. Os dados foram coletados mediante aplicação de questionário a 11 profissionais de enfermagem e de observação não participante, analisados segundo a Análise de Conteúdo de Bardin. Resultados: Identificaram-se quatro categorias de conteúdo: (1) o conhecimento da equipe sobre as etapas do tratamento quimioterápico e percepções gerais acerca da segurança dos pacientes; (2) higienização das mãos e risco de queda; (3) inexatidão na identificação do paciente e na comunicação eficaz; e (4) administração segura de quimioterapia antineoplásica. Nessas categorias, foi possível constatar falhas de conhecimento nas etapas do tratamento e administraçáo segura de quimioterapia antineoplásica, na identificação do paciente e na comunicação eficaz. Observou-se o empenho dos profissionais do serviço em implementar o Programa Nacional de Segurança do Paciente (PNSP) com a identificaçáo dos pacientes com crachá, prescriçóes impressas e protocolos de extravasamento e derramamento. Entretanto, a adesão a higienização das mãos não foi observada. Conclusão: Inferiu-se a necessidade de educação permanente e continuada dos profissionais, apesar da existência de itens assistenciais que atendem aos protocolos do PNSP.

Palavras-chave: Enfermagem Oncológica; Conhecimento; Assistência Ambulatorial; Antineoplásicos; Segurança do Paciente.

\section{Abstract}

Introduction: Patient safety is becoming increasingly important at the national and international levels, especially in oncology, where there is a growing concern about the errors/adverse events related to antineoplastic chemotherapy drugs, with the main premise being the quality of health care and care safe. Objective: To evaluate the knowledge of nursing professionals regarding the safety of cancer patients undergoing chemotherapy. Method: A qualitative study, carried out at the outpatient clinic of a High Complexity Oncology Care Unit, located in the State of Pará. Data were collected through a questionnaire to 11 non-participating nursing professionals, analyzed according to the analysis of Bardin content. Results: Four categories of content were identified: (1) the team's knowledge about the steps of chemotherapy treatment and general perceptions about patient safety; (2) hand hygiene and risk of falling; (3) inaccuracy in patient identification and effective communication; and (4) safe administration of antineoplastic chemotherapy. In these categories it was possible to verify knowledge gaps in the stages of the treatment and safe administration of antineoplastic chemotherapy, in the identification of the patient and in effective communication. It was observed the commitment of service professionals to implement the National Patient Safety Program (NPSP) with the identification of patients with badges, printed prescriptions and protocols of extravasation and spill. However, adherence to hand hygiene was not observed. Conclusion: The need for continuing and continuing education of professionals was inferred, despite the existence of care items that comply with the protocols of the NPSP.

Key words: Oncology Nursing; Knowledge; Ambulatory Care; Antineoplastic Agents; Patient Safety.

\section{Resumen}

Introducción: La seguridad del paciente es cada vez más importante a nivel nacional e internacional, especialmente en oncología, en el cual existe una creciente preocupación por los errores/eventos adversos relacionados con los medicamentos antineoplásicos para quimioterapia, cuya principal premisa es la calidad de la atención médica y la atención segura. Objetivo: Evaluar el conocimiento de los profesionales de enfermería sobre la seguridad de los pacientes con cáncer que se someten a quimioterapia. Método: un estudio cualitativo, realizado en la clínica ambulatoria de una Unidad de Atención Oncológica de Alta Complejidad, ubicada en el estado de Pará. Los datos fueron recolectados a través de un cuestionario a 11 profesionales de enfermería nos participantes, analizados de acuerdo con el contenido del análisis de Bardin. Resultados: Se identificaron cuatro categorías de contenido: (1) el conocimiento del equipo sobre los pasos del tratamiento de quimioterapia y las percepciones generales sobre la seguridad del paciente; (2) higiene de manos y riesgo de caerse; (3) inexactitud en la identificación del paciente y la comunicación efectiva; y (4) la administración segura de quimioterapia antineoplásica. En estas categorías fue posible verificar las brechas de conocimiento en las etapas del tratamiento y la administración segura de la quimioterapia antineoplásica, en la identificación del paciente y en la comunicación efectiva. Se observó el compromiso de los profesionales de servicio para implementar el Programa Nacional de Seguridad del Paciente (PNSP) con la identificación de pacientes con credenciales, prescripciones impresas y protocolos de extravasación y derrame. Sin embargo, no se observó adherencia a la higiene de las manos. Conclusión: Se infirió la necesidad de educación permanente y continuada de los profesionales, a pesar de la existencia de elementos de atención que cumplen con los protocolos del PNSP. Palabras clave: Enfermería Oncológica; Conocimiento; Atención Ambulatoria; Antineoplásicos; Seguridad del Paciente.

1 Universidade Federal do Pará (UFPA). Belém (PA), Brasil. Orcid iD: https://orcid.org/0000-0002-1820-5771

2 UFPA. Belém (PA), Brasil. Orcid iD: https://orcid.org/0000-0003-3025-1145

${ }^{3}$ UFPA. Belém (PA), Brasil. Orcid iD: https://orcid.org/0000-0002-2506-1622

${ }^{4}$ UFPA. Belém (PA), Brasil. Orcid iD: https://orcid.org/0000-0001-8468-2405

${ }^{5}$ UFPA. Belém (PA), Brasil. Orcid iD: https://orcid.org/0000-0002-3571-734

${ }^{6}$ UFPA. Belém (PA), Brasil. Orcid iD: https://orcid.org/0000-0002-8154-0310

${ }^{7}$ UFPA. Belém (PA), Brasil. Orcid iD: https://orcid.org/0000-0001-8812-2923

Endereço para correspondência: Aline Gonçalves da Costa. Travessa Primeira de Queluz, 255 - Belém (PA), Brasil. CEP 66090-520. E-mail: gc_aline@hotmail.com 


\section{INTRODUÇÃO}

A qualidade do cuidado em saúde, relacionado à segurança do paciente, vem adquirindo grande importância em níveis nacional e internacional, tendo como principal objetivo a assistência segura. Dessa forma, a Organização Mundial da Saúde (OMS) criou a World Alliance for Patient Safety (Aliança Mundial pela Segurança do Paciente), que visa a organizar os conceitos e as definiçóes sobre a segurança do paciente e propor medidas para reduzir os riscos de eventos adversos ${ }^{1}$.

No Brasil, foi instituído pela Portaria GM/MS no. 529/2013 o Programa Nacional de Segurança do Paciente $(\mathrm{PNSP})^{2}$, com o intuito de contribuir para a qualificação do cuidado em saúde em todos os estabelecimentos de saúde do território nacional. Sendo assim, por meio da Portaria GM/MS no. 1.377, de 9 de julho de 2013, e da Portaria no. 2.095, de 24 de setembro de 2013, aprovaram os protocolos básicos de segurança do paciente como instrumentos para implantação das açóes em segurança do paciente, a saber: (i) identificação do paciente; (ii) prevenção de úlcera por pressão; (iii) segurança na prescrição, uso e administração de medicamentos; (iv) cirurgia segura; (v) prática de higiene das mãos em serviço de saúde; e (vi) prevenção de quedas. Esses protocolos dão apoio à implantação do PNSP e à implementação de protocolos, guias e manuais de segurança do paciente, além de estímulo à notificação e análise dos eventos adversos ocorridos ${ }^{1}$.

Em 2008, houve a criação da Rede Brasileira de Enfermagem e Segurança do Paciente (Rebraensp) vinculada à Rede Internacional de Enfermagem e Segurança do Paciente da Organização Pan-Americana da Saúde (Opas), composta por enfermeiros das áreas de ensino, assistência, gestão e pesquisa, de instituiçóes públicas e privadas. Entre as metas da rede, destacam-se a difusão dos preceitos básicos da segurança do paciente; identificação de pontos críticos para a segurança do paciente; e desenvolvimento de ferramentas adequadas (guias de prática clínica, grupos de análise de eventos adversos e simulaçóes) ${ }^{3}$.

Ademais, para que a segurança do paciente aconteça de fato, a cultura de segurança precisa estar estruturada nas instituiçóes, com o estabelecimento de um processo de comunicação adequado, confiança, aprendizado organizacional, comprometimento coletivo em relação aos aspectos da segurança, liderança, importância da temática e abordagem não punitiva ao erro ${ }^{4}$.

Há uma preocupação crescente com os erros no âmbito da oncologia em relação aos quimioterápicos por causa dos efeitos causados pelo seu uso, que, por vezes, pode ser fatal. Isso porque os medicamentos antineoplásicos apresentam um baixo índice terapêutico; ou seja, a dose letal é muito próxima da dose terapêutica. Além de estreita margem de segurança, tornando-os medicamentos perigosos e exigindo uma atençáo redobrada dos profissionais envolvidos no seu processo de prescriçáo, preparo, dispensação e administraçãoo ${ }^{5-7}$.

Dados atuais revelam que o quantitativo de óbitos decorrentes dos eventos adversos em saúde é alarmante ${ }^{4}$. Esses eventos, em uma ou mais etapas do processo de assistência, como a realização de administração de medicamento, podem ter consequências graves e levar o paciente a óbito ${ }^{8}$. Sendo assim, destaca-se que o papel do enfermeiro oncológico envolve a aplicação das práticas e padróes de enfermagem oncológica da Oncology Nurse Society (NOS) e da American Nurse Association (ANA), desenvolvidos para medir e avaliar uma assistência holística e de qualidade. Para isso, faz-se necessária a qualificação da equipe de enfermagem em virtude das particularidades do mecanismo de ação dos medicamentos, além das possíveis toxicidades decorrentes do tratamento ${ }^{9,10}$.

Diante disso, compreende-se a importância de investigar se os profissionais de enfermagem que atuam na oncologia acompanham as atualizaçôes e os avanços científicos relacionados à segurança do paciente. Nesse sentido, definiu-se, como objetivo, avaliar o conhecimento dos profissionais de enfermagem quanto à segurança do paciente oncológico em tratamento quimioterápico, sendo traçado em consonância com o PNSP instituído pelo Ministério da Saúde.

\section{MÉTODO}

Esta pesquisa caracteriza-se como qualitativa, exploratória e descritiva. O campo de estudo foi o serviço de quimioterapia antineoplásica da Unidade de Atendimento de Alta Complexidade em Oncologia (Unacon) de um hospital de ensino, habilitado para a atenção especializada em Oncologia no Norte do Brasil, no período de janeiro a março de 2018.

O estudo recrutou os 12 profissionais da equipe de enfermagem da unidade de atendimento oncológico (6 técnicos de enfermagem e 6 enfermeiros), conforme os seguintes critérios de inclusão: (i) profissionais da área da enfermagem; (ii) aqueles envolvidos nas etapas de tratamento ambulatorial de quimioterapia antineoplásica; e (iii) que aceitaram participar do estudo ao assinar o Termo de Consentimento Livre e Esclarecido (TCLE). Já os critérios de exclusão foram: aqueles que não aceitaram participar da pesquisa, que não sejam da área de enfermagem e não estejam no quadro de profissionais atuantes no ambulatório de quimioterapia. Após as exclusôes, a populaçẫo foi constituída por 11 
profissionais de enfermagem (6 técnicos de enfermagem e 5 enfermeiros).

A coleta de dados ocorreu em duas etapas. Na primeira, utilizou-se um questionário de pesquisa, criado e baseado nos preceitos sobre segurança do paciente conforme o PNSP/2013. Para a aplicação do questionário, foi realizado contato prévio com os profissionais, para que a pesquisadora fornecesse informaçôes referentes ao objetivo do estudo, técnica de coleta de dados e aspectos éticos.

O questionário continha perguntas fechadas referentes ao sexo, categoria profissional, tempo de formação e de trabalho na instituição e turno de trabalho; ou seja, dados que possibilitassem traçar um perfil das amostras e perguntas abertas acerca da assistência aos pacientes em quimioterapia antineoplásica, como: orientaçôes e cuidados fornecidos, etapas da quimioterapia, riscos de exposição na administração de quimioterapia antineoplásica e sinais e sintomas relacionados à aplicação.

Foi disponibilizado um período de 24 horas para responder e entregar os questionários, uma vez que o número de profissionais era reduzido e havia grande demanda de pacientes, não houve possibilidade de a primeira etapa ser aplicada em forma de formulário durante o horário de serviço, nem em concomitância com a segunda etapa.

A segunda etapa foi realizada durante observaçóes in loco e aplicação de roteiro estruturado (checklist). Essa etapa objetivou observar as fases do tratamento com quimioterapia antineoplásica in loco, em seus vários momentos: desde o recebimento do paciente, a conferência da prescrição do paciente, a adequação dos equipamentos de proteção individuais (EPI), a chegada dos quimioterápicos provindo da farmácia, o acondicionamento, a administraçáo do medicamento, o monitoramento e o registro dos medicamentos administrados até o descarte dos materiais envolvidos no processo. A amostragem foi por conveniência em virtude de escala fixa da equipe que assistia aos pacientes, sendo realizadas dez observaçóes que perfizeram um total de 30 horas; foram acompanhadas quatro técnicas de enfermagem e uma enfermeira.

A análise de conteúdo temática de Bardin ${ }^{11}$ subsidiou a análise dos dados dos questionários, visando à interpretaçáo de material de caráter qualitativo, assegurando uma descrição objetiva, sistemática e com a riqueza manifesta expressa neles. Os dados foram organizados e categorizados em torno de três polos: a) pré-análise, leitura inicial sem julgamentos ("naive"); b) exploraçáo do material (análise estrutural e categorização do conteúdo); e c) o tratamento dos resultados (inferência) com interpretação crítica e discussão.

Para garantir o anonimato dos sujeitos e dos resultados, eles foram codificados com a sigla ENF para os enfermeiros e TE para os técnicos de enfermagem, seguidos do número do questionário. Essa escolha se deu levando-se em consideração as siglas das categorias profissionais indicadas na Resoluçáo do Conselho Federal de Enfermagem (Cofen) no ${ }^{\circ} .545$ de $2017^{12}$.

Este estudo seguiu os preceitos éticos vigentes e foi aprovado pelo Comitê de Ética em Pesquisa do Núcleo de Pesquisa em Oncologia da Universidade Federal do Pará, sob número de parecer 2.440.663/2017 e da emenda ${ }^{\circ}$. 2.562.304/2017.

\section{RESULTADOS}

O gênero feminino prevaleceu em $100 \%$ da amostra do estudo. Entre as 11 profissionais, cinco $(45,4 \%)$ eram enfermeiras, todas especialistas em enfermagem oncológica e com atualização em cursos relacionados ao assunto; quatro enfermeiras (80\%) possuíam também o mestrado. O tempo de formaçáo foi acima de 12 anos, com aproximadamente seis anos de atuação no serviço.

As outras seis profissionais eram técnicas de enfermagem $(54,6 \%)$; o tempo de formação foi de quatro a 12 anos ou mais, com tempo de atuação em torno de três a seis anos. Três (50\%) não receberam treinamento antes de iniciar no serviço de quimioterapia antineoplásica, mas afirmaram ter participado de cursos de atualizaçáo em oncologia.

Com relação ao processo de categorização dos dados, emergiram quatro categorias, as quais são apresentadas em paralelo com a observação não participante e direta da assistência aos pacientes oncológicos em tratamento ambulatorial com quimioterapia antineoplásica.

\section{CATEGORIA 1: 0 CONHECIMENTO DA EQUIPE SOBRE AS ETAPAS DO TRATAMENTO QUIMIOTERÁPICO E PERCEPÇÕES GERAIS ACERCA DA SEGURANÇA DOS PACIENTES}

Ao analisar as respostas acerca das etapas do tratamento com quimioterapia antineoplásica por meio da pergunta "Quais são as etapas obrigatórias do tratamento com quimioterapia antineoplásica?”, foi possível verificar que a maioria dos participantes possuía conhecimento fragmentado ou incompleto sobre o questionamento. Como pode ser observado a seguir.

Prescrição médica, agendamento, avaliação das prescriçôes, manipulação, conferência, administração, registros e descarte (ENF3).

Após a biópsia, o paciente é encaminhado para consulta com o médico oncologista e depois disso poderá ser encaminhado para quimioterapia ou cirurgia (TE2).

Importante destacar, também, que uma enfermeira não soube responder (deixou em branco) o questionamento 
sobre as etapas da quimioterapia antineoplásica e uma citou apenas as fases de administração do quimioterápico, como se mostra a seguir:

Pré-quimioterapia, quimioterapia e pós-quimioterapia (ENF4).

Com relação à indagação "A que riscos os pacientes em tratamento quimioterápico estão expostos?”, as afirmaçōes dos profissionais foram as seguintes:

Extravasamento/derramamento, contaminação local, reaçôes adversas (ENF5).

Extravasamento, reação alérgica e derramamento (TE5).

Com isso, foi possível perceber ainda que as profissionais estavam atentas aos riscos não modificáveis, como o comprometimento da rede venosa. Atençáo essa que é citada na resposta à pergunta "Qual(is) cuidado(s) de enfermagem deve(m) ser tomado(s) na utilizaçáo de uma via endovenosa para a administraçáo de medicamentos antineoplásicos?”.

Dar preferência a punção de veias de grosso calibre e em membros superiores (MMSS), evitar áreas de articulação, não puncionar no braço do mesmo lado da mastectomia. Ficar sempre atento durante a administração da quimioterapia e parar a infusão ao suspeitar de extravasamento. Sempre "lavar" a veia após a infusấo de cada quimioterapia [...] (ENF1).

[...] evitar administração de quimioterapia em veias periféricas com escalpe. Fixar a punção venosa, observar a presença de sinais flogísticos. Evitar puncionar veias do mesmo lado de aplicaçáo de radioterapia e do mesmo lado de mastectomia (TE1).

Já em relação às intervençôes mencionadas quando questionadas quanto à "Quais medidas são tomadas em caso de extravasamento ou derramamento de quimioterápicos?”. Destacou-se:

Extravasamento: interromper a infusáo, sem retirar o cateter venoso, aspirar o conteúdo extravasado, retirar o cateter venoso, se droga vesicante aplicar compressa quente ou gelada de acordo com a medicação. Pode-se administrar $1 \mathrm{~mL}$ de dexametasona em leque no local extravasado. Derramamento: o local deve ser isolado, deve-se paramentar antes de iniciar o procedimento (com luva, máscara, gorro). Se derramamento de líquido, limpar com compressas secas; se for pó, as compressas devem ser úmidas. Registrar o ocorrido (ENF1).

Interromper infusão. Fazer uso de protocolo da instituição: kit de extravasamento/kit de derramamento (ENF3).

No que se refere às "percepçôes gerais da equipe de Enfermagem sobre a segurança do paciente", as profissionais relacionaram os riscos apenas ao paciente e subjugaram os riscos ocupacionais. Como se conclui a seguir:

Se aplicadas todas as medidas, protocolos e cuidados, conseguimos que a aplicação de terapia antineoplásica se dê da forma mais tranquila possível, com todos os benefícios para o paciente (ENF1).

\section{CATEGORIA 2: HIGIENIZAÇÃO DAS MÃOS E RISCO DE QUEDA}

Entre as condutas relatadas pelas profissionais, observou-se que nenhuma das participantes citou a higienização das mãos como procedimento de assistência ao paciente em quimioterapia, sendo tal conduta corroborada durante realizaçáo de roteiro observacional. Nesse sentido, em todos os processos assistenciais observados, nenhuma profissional higienizou as mãos, nem antes nem após contato com o paciente; ou seja, durante realização de punção, verificação de sinais vitais, instalação de infusão de quimioterapia antineoplásica e contato com imediaçôes próximas ao paciente.

Destaca-se, ainda, a ausência de avaliação do risco de queda durante a assistência ao paciente oncológico, náo sendo aplicada nenhuma escala para avaliar a presença de fatores de risco contribuintes adequados ao perfil de cada paciente. Apesar disso, os profissionais descreveram alguns cuidados quando responderam à pergunta "Quais os cuidados com pacientes acamados ou com mobilidade prejudicada e/ou reduzida durante o tratamento quimioterápico ambulatorial? É aplicada a escala de Morse?", tais quais:

Auxiliar o paciente a ir ao banheiro, orientar o acompanhante no auxílio ao paciente, transportar o paciente em cadeira de rodas. Acomodar o paciente no leito com proteção lateral ou em poltrona próxima ao banheiro. Não utilizamos a escala de avaliação (ENF1).

Ajudá-lo na sua mobilidade e conforto (TE3).

Além dos cuidados relatados, na observação não participante, foi possível perceber que os pacientes que apresentavam fatores de risco para queda eram posicionados 
em leitos próximos ao posto de enfermagem, bem como foi permitido a permanência de um acompanhante durante a sessão de quimioterapia, o que fortalecia as medidas de segurança para pacientes oncológicos com risco de queda.

Ademais, observou-se que as profissionais de enfermagem realizavam orientaçôes aos familiares e pacientes sobre medidas preventivas de quedas quando os pacientes chegavam ao ambulatório de quimioterapia antineoplásica.

\section{CATEGORIA 3: INEXATIDÃO NA IDENTIFICAÇÃO DO PACIENTE E NA COMUNICAÇÃO EFICAZ}

Conforme eram as respostas à pergunta "Como é feita a identificação do paciente no ambulatório de quimioterapia?", verificou-se que a identificação dos pacientes se dava por meio de crachás contendo nome completo e matrícula. Por outro lado, no decorrer da observação não participante, não foi possível verificar a confirmação subjetiva da identificação do paciente antes da administração de quimioterapia antineoplásica.

Quanto às indagaçóes "Você orienta os pacientes sobre o medicamento que ele receberá? Se sim, que informaçôes são fornecidas?" e "Em sua opinião o que é importante para que se desenvolva um bom vínculo entre profissionais-paciente? Essa relação influencia na adesão do paciente ao tratamento quimioterápico?", destacaram-se as seguintes respostas:

Sim. Oriento sobre as possíveis reaçôes e o que poderá acontecer, como: alopecia, náuseas, vômitos, diarreia, não poder pegar sol, cuidados com alimentação, tomar bastante líquido (TE6).

Para desenvolver um bom vínculo, é necessário empatia, diálogo, linguagem acessível. Influência sim na adesão do paciente (ENF5).

Comprovou-se, ainda, durante observação não participante, que todas as profissionais ofereceram orientações acerca dos cuidados com a via de infusão e os sinais e sintomas de extravasamento ou infiltração de quimioterapia antineoplásica aos pacientes e familiares.

No que se refere à comunicação entre as profissionais quando questionadas sobre "Você já observou algum erro na prescrição de quimioterapia antineoplásica? Se sim, quais e qual foi sua conduta?", detectou-se que havia trocas de informaçôes por meio da verbalizaçáo.

Comuniquei ao médico de plantão [erro na prescrição de quimioterapia antineoplásica] e à farmácia para correção do erro (ENF1).
Informei à enfermeira do plantão [troca de rótulos com o nome do paciente] (TE3).

Ressaltando-se, entretanto, que algumas profissionais perceberam equívocos na comunicação escrita (prescrição de quimioterapia antineoplásica), mas não foi possível identificar se alguma conduta foi tomada:

Nas prescrições, vem fenergan endovenoso, sendo que é intramuscular (TE5).

\section{CATEGORIA 4: AdMINISTRAÇÃO SEGURA DE QUIMIOTERAPIA ANTINEOPLÁSICA}

Evidenciou-se, por meio da pergunta "Que informaçóes são encontradas nas prescriçóes dos medicamentos antineoplásicos?”, que na instituição as prescriçôes são digitadas e impressas, além de conterem informaçóes fundamentais para prevenção de erros de medicação, como:

Nome do paciente, matrícula, idade, peso, altura, índice de massa corporal, dose da medicaçáo, tempo de infusão, dia, ciclo, nome do médico (ENF1).

Medicaçáo prescrita, protocolo, ciclo, nome do paciente, matrícula, idade, data, o tipo de câncer e observação para checar exames (TE6).

Já em relação ao questionamento "Quais procedimentos são realizados antes de iniciar a administração de quimioterapia antineoplásica?”, destacou-se:

Aferição dos sinais vitais, avaliação da performance status e hemograma, se necessário $(\mathrm{S} / \mathrm{N})$. Punçáo venosa periférica ou Port-a-cath, quimioterapia endovenosa (quimioterapia endovenosa). Comprovar a permeabilidade do acesso venoso antes da administração da quimioterapia (se quimioterapia endovenosa). Checar o nome do paciente na prescrição e conferir o rótulo da quimioterapia com os dados da prescriçáo (droga, diluição, tempo de infusão etc.) (ENF1)

É realizada punção do acesso venoso periférico, preparo e administração de medicaçóes préquimioterapia (TE2).

Convém salientar que, ao longo da observação não participante, apenas as profissionais técnicas se certificaram se o acesso venoso estava pronto para uso (pérvio) e conferiram se os medicamentos entregues pela farmácia eram os corretos. Além disso, as principais vias de acesso utilizadas para tratamento com quimioterapia antineoplásica foram o acesso venoso periférico, via oral e acesso central totalmente implantado (Port ou Port-a-cath). 
Constatou-se, também, que a conferência de nome, dose, horário e via de administração no rótulo da quimioterapia antineoplásica foi realizada comparando-se apenas com a prescrição do medicamento, contudo, o paciente era informado do início da infusão. Outro aspecto a ser considerado é que todos os pré-quimioterápicos prescritos foram administrados adequadamente antes do início da quimioterapia antineoplásica, passo importante para a eficiência da droga e eficácia do tratamento. Evidenciou-se, ainda, a verificação dos sinais vitais e eventuais queixas dos pacientes.

As respostas seguintes demonstraram a forma como as profissionais realizavam o registro da administraçáo de quimioterapia antineoplásica no prontuário do paciente, quando emergiu o questionamento "Como você realiza o registro da administração da medicaçáo antineoplásica no prontuário do paciente?":

Por meio de evoluçáo contendo ciclo, medicação, estado atual [PS: performance status], possíveis reaçôes, sinais vitais e preenchimento de demais formulários de segurança do paciente (ENF2).

Checo o horário de início e término de infusão. A localização da punçáo venosa, o tipo de cateter utilizado (jelco, nexiva etc). Anoto os valores dos sinais vitais e possíveis intercorrências (TE1).

Com relação aos registros assistenciais, no ínterim da observação não participante, constatou-se que as enfermeiras realizavam as anotações de enfermagem no prontuário dos pacientes, já as técnicas de enfermagem faziam as anotaçōes no verso das prescrições, sendo registrado o nome do medicamento administrado, a dosagem, horário, sinais vitais, a via de administração e as reaçōes adversas, caso ocorressem.

Inferiu-se a associação do "fazer a checagem/avaliação da prescrição" ao processo de registro/anotação após a administração de quimioterapia antineoplásica, quando as profissionais responderam à pergunta "Quem deve fazer a avaliação/supervisão da prescrição da medicação antineoplásica?”. Como se pode deduzir em seguida:

Quem administra (TE4).

Técnico de enfermagem e enfermeiro dependendo da medicação (TE2).

Identificou-se que alguns profissionais possuíam dificuldades em diferenciar antineoplásicos vesicantes e irritantes, quando questionados acerca de "O que é um medicamento antineoplásico vesicante ou irritante?". Das $11(100 \%)$ profissionais, sete $(63,7 \%)$ erraram ou confundiram (cinco técnicas de enfermagem e duas enfermeiras) ao responder o que são medicamentos vesicantes, porém conseguiram distinguir que as reaçôes são mais intensas do que as provocadas pelas drogas irritantes.

Vesicantes são drogas que provocam irritação severa [...]. Irritantes são drogas que causam irritaçáo cutânea (ENF3).

Antineoplásico vesicante, se extravasa, pode ocorrer um dano maior do que o medicamento irritante (TE2)

Notou-se que todos os profissionais souberam descrever os equipamentos de proteçáo que devem ser utilizados na medida em que as respostas à questáo foram analisadas: "Quais equipamentos de proteção são utilizados no manuseio de quimioterápicos antineoplásicos (preparo, administração, descarte de resíduos e excretas dos pacientes)?”. Destacando-se:

Gorro, óculos, capote maga longa impermeável, luvas, sapatos fechados [administração]. Capela de fluxo laminar, máscara [preparo] (ENF2).

Avental, luvas de procedimento, óculos de proteção, gorro (TE6).

Apesar dessas colocações, no decurso da observação não participante, os profissionais usavam roupas específicas do setor com mangas curtas; a máscara PFF2 com filtro foi usada somente no transporte (por isopor) da farmácia até a sala de quimioterapia; instalação e descarte dos quimioterapia antineoplásica. Já durante a administração dos pré-quimioterápicos, utilizaram máscara cirúrgica descartável com elástico.

Importante frisar, também, que o transporte, administração e descarte de quimioterapia antineoplásica foram realizados por profissional técnico, mas a solicitação dos medicamentos à farmácia era feita pela enfermeira do plantão, no entanto, não era ela quem fazia a conferência das drogas.

Quanto à forma de evitar erros na administração de quimioterapia antineoplásica, a resposta que prevaleceu foi a seguinte:

Seguir os nove certos na administraçáo de medicamentos (TE1).

\section{DISCUSSÃO}

A chamada feminização da força de trabalho é destacada como uma das tendências em foco: expansão 
da capacidade instalada, municipalização dos empregos, ambulatorização dos atendimentos, maior qualificação das equipes e flexibilidades dos vínculos. Considerando-se a história da enfermagem, nesse contexto, como a primeira profissão universitária feminina no $\mathrm{Brasil}^{13}$, a total prevalência de profissionais do sexo feminino no estudo foi um dado esperado.

Mesmo assim, salienta-se que a falta de treinamento de metade dos profissionais técnicos do estudo é um dado preocupante e também encontrado em outros estudos realizados ${ }^{14-16}$, uma vez que os profissionais de enfermagem sáo o último elo na cadeia de prestadores de cuidados de saúde no tratamento com medicamentos quimioterápicos. Essa falta de capacitação específica pode elevar os riscos de ocorrência de erros e eventos adversos, além de expor a saúde ocupacional ${ }^{17}$.

Desse modo, seguir as diretrizes da Sociedade Americana de Farmacêuticos do Sistema de Saúde (ASHP), como requisito para o credenciamento da enfermagem com medicamentos quimioterápicos, garante a sustentaçáo e o funcionamento seguro do serviço de quimioterapia antineoplásica, tais como: políticas e procedimentos organizacionais que exijam funcionários de enfermagem com treinamento concluído; demonstração de competência na administração de medicamentos intravenosos; e reavaliação organizacional periódica dos funcionários ${ }^{7}$.

Há que se ressaltar que conhecer riscos modificáveis relacionados ao uso de quimioterapia antineoplásica, como extravasamentos de medicaçóes, comprometimento dos vasos sanguíneos e escape de drogas, é fundamental para evitar reaçôes agudas, bem como reduzir os efeitos psicológicos associados à ocorrência de eventos adversos ou acidentes ${ }^{18}$.

Somado a isso, as medidas citadas para a prevenção do extravasamento ou derramamento de quimioterapia antineoplásica estão em concordância com as encontradas na literatura ${ }^{19}$, apesar de essa competência ser delegada às técnicas de enfermagem na instituição pesquisada. Convém ressaltar que a administração de quimioterapia antineoplásica é de responsabilidade do enfermeiro, conforme prática regulamentada na Resolução n ${ }^{\circ} .569$ de 2018 do Cofen $^{20}$. O enfermeiro deve, ainda, supervisionar sua equipe e promover ações de educação permanente, a fim de excluir possíveis riscos ocupacionais.

Também foram levantadas análises referentes às percepçóes de segurança ocupacional, como risco de exposição às drogas e à possível contaminação do ambiente de trabalho, além dos fatores ergonômicos e psicossociais. As respostas das profissionais sugeriram déficit de conhecimento no assunto, tal como encontrado no estudo de Senna et al. ${ }^{21}$. Essas constatações apontam para a necessidade de dar importância ao treinamento da equipe para protegê-los de efeitos tóxicos quando na exposição a quimioterapia antineoplásica e seus resíduos durante o preparo, administraçáo e descarte, tendo em vista que essas drogas são conhecidas por serem mutagênicas, carcinogênicas e teratogênicas ${ }^{22-24}$.

Quanto à não higienização das mãos pelos profissionais, a conduta vai de encontro ao protocolo instituído pelo Ministério da Saúde ${ }^{25}$, concernente à medida de higienização das mãos, a qual deve ser aplicada em todos os pontos de assistência (paciente, profissional de saúde e assistência ou tratamento direto ao paciente ou a suas imediaçôes), visando à segurança do paciente, dos profissionais de saúde e de todos aqueles envolvidos nos cuidados aos pacientes.

No que se refere aos EPI, observou-se que o vestuário e o uso da máscara PFF2 não estavam de acordo com a RDC no. 220 de 2004. Estudos mostram que o uso de EPI é ignorado pela maioria dos profissionais de enfermagem que atuam com drogas quimioterápicas, isso indica a importância do desenvolvimento de programas educacionais que motivem a equipe a usar EPI para superar essa fragilidade no conhecimento. Este estudo demonstrou, ainda, que a educação on-line pode ser eficaz como meio de promover práticas seguras em conformidade com o uso de $\mathrm{EPI}^{26}$.

No tocante aos fatores de risco de queda, há estudos que mostram que hospitais oncológicos apresentam incidência de queda em torno de $18 \%$ acima dos hospitais gerais, em virtude de os pacientes oncológicos, em sua maioria, apresentarem condiçóes nutricionais mais frágeis e fazerem uso de medicações analgésicas potentes para controle e alívio da dor, aspectos esses que os predispóem a um risco maior às quedas. Estratégias como o desenvolvimento de um protocolo de prevenção de quedas no qual se estabeleça critérios para reconhecer precocemente aqueles pacientes que apresentam maior risco reforçam as barreiras de prevenção às quedas, mesmo em pessoas em atendimento ambulatorial ${ }^{27}$.

Para evitar esses eventos, a Rebraensp e o Ministério da Saúde recomendam um programa de avaliação do risco de quedas relacionado à pessoa e ao ambiente, utilização de escalas de avaliação universais de risco (escala de Morse); triagem ou avaliaçáo para individualizar o cuidado; uso de linguagem acessível para informaçáo e orientaçáo ao paciente e acompanhante; criação de um indicador institucional de quedas a partir de um sistema de notificaçấo e gerenciamento desses eventos adversos ${ }^{28}$; além da criação de um ambiente de cuidado seguro, como: pisos antiderrapantes; mobiliário, iluminaçóes adequadas, vestuário e calçados adequados; e movimentação segura dos pacientes ${ }^{29}$. Essas recomendaçóes visam a uma 
prevenção primária contínua para ser eficaz e eficiente na proteção aos pacientes oncológicos diante de riscos de queda.

Já a identificação correta do paciente busca evitar ou reduzir a ocorrência de incidentes e assegurar o cuidado ou tratamento ao paciente correto, incluindo três intervençóes: (I) identificar o paciente; (II) educar o paciente, acompanhante, familiar ou cuidador; e (III) confirmar a identificaçâo do paciente antes do cuidado ${ }^{30}$.

Ressalta-se que, na instituição pesquisada, a identificação dos pacientes está de acordo com os identificadores (no mínimo dois) que devem ser utilizados na intervenção I preconizada, como: nome completo do paciente; nome completo da máe do paciente; data de nascimento do paciente; e número de prontuário do paciente ${ }^{30}$. No entanto, as intervençóes II e III não foram observadas. Para atendê-las, recomenda-se que o profissional responsável pelo cuidado pergunte o nome ao paciente, familiar ou acompanhante e confira as informaçóes contidas na identificaçáo do paciente com o cuidado/tratamento prescrito, ou com a rotulagem do material que será utilizado, mesmo que o profissional de saúde conheça o paciente, para garantir que ele receba o tratamento correto $^{30}$.

No levantamento da importância do processo comunicativo profissional-paciente, observou-se que a comunicação referida pelas profissionais técnicas aos pacientes não vinha, aparentemente, acompanhada de uma preocupação na forma como era comunicada, uma vez que as profissionais não se certificaram do entendimento das orientaçôes repassadas. Assim, a Rebraensp ${ }^{28}$ sugere, como aspecto fundamental para uma comunicação eficaz, o entendimento ou compreensão significativa por parte do receptor (paciente, acompanhante, familiar) da informação repassada ou pretendida pelos profissionais. Nessa perspectiva, para que as informaçóes clínicas sejam transmitidas consistentemente, são necessários atitudes, habilidades e conhecimentos da equipe e, principalmente, uma comunicação adequada ${ }^{31}$.

Outrossim, a comunicação pouco eficaz entre profissionais também pode repercutir no cuidado e contribuir para desfechos negativos ${ }^{32}$. Logo, recomenda-se que exista avaliação do processo de comunicação do serviço de saúde, identificando os pontos críticos que possam levar à ocorrência de eventos adversos.

Quanto ao processo de administração de quimioterapia antineoplásica, além do processo de administração propriamente dita citado pelas profissionais, abarca-se também o transporte, o descarte e o registro ${ }^{33}$. Desse modo, salienta-se a adequaçáo da prescrição segura de medicamentos realizada pela instituição, que inclui: identificação do paciente com no mínimo nome completo e data de nascimento; identificação do prescritor (nome, registro de classe e assinatura); data de validade; e legibilidade ${ }^{34}$. Destaca-se, porém, a ausência da dupla checagem/avaliação (farmacêutico e enfermeiro) ${ }^{32}$ antes do preparo e da administração da prescrição médica.

Convém ressaltar que os registros de enfermagem são elementos imprescindíveis ao processo de cuidado e, quando redigidos de maneira que retratem a realidade a ser documentada, tal como analisado no estudo, possibilitam a comunicaçáo efetiva e o respaldo legal aos profissionais e serviços de saúde. Admite-se ainda citar que os registros consistem no mais importante instrumento de avaliação da qualidade de atuação da enfermagem, representando $50 \%$ das informaçōes inerentes ao cuidado dos pacientes registradas no prontuário ${ }^{35}$.

É necessário frisar que todo profissional de saúde, ao administrar um medicamento, deve sempre checar os "nove certos": medicamento certo, dose certa, via certa, horário certo, paciente certo, registro certo, ação certa, forma certa e resposta certa $^{36}$. Essa conduta melhora a segurança e a qualidade da assistência prestada ao paciente ${ }^{37}$.

Evidências sugerem, portanto, que a implementação de padróes e processos de segurança ajudam prevenir erros de administração de quimioterapia antineoplásica; entre eles, o conhecimento dos tipos de drogas, o qual melhora a segurança do paciente e garante a prestação de cuidados mais seguros ${ }^{34}$.

\section{CONCLUSÃO}

Os resultados da pesquisa apontaram fragilidades no conhecimento dos profissionais de enfermagem para uma assistência segura ao paciente oncológico, por não perceberem a exposição aos riscos ocupacionais no ambulatório de quimioterapia antineoplásica quando negligenciam itens fundamentais de biossegurança, como a higienização das mãos e o uso adequado de EPI, além de pouco envolvimento nas orientaçóes fornecidas ao paciente ou familiar quando deve ser esclarecido tudo o que seja relevante para um tratamento bem-sucedido. Contudo, destaca-se o empenho da equipe de enfermagem em atender aos protocolos de segurança do paciente do PNSP com a identificação dos pacientes com crachá, uso de prescriçốes eletrônicas e desenvolvimento de protocolos de extravasamento e derramamento.

Entende-se, portanto, a importância da implementação de sistemas padronizados para facilitar uma administração mais segura de quimioterapia antineoplásica. Para isso, se faz necessária a educação permanente dos profissionais que atuam na assistência aos pacientes oncológicos, realizando oficinas educativas, palestras e momentos de discussão 
sobre a tema, para que desenvolvam uma cultura de segurança no serviço de quimioterapia oncológica.

Como contribuiçãa desta pesquisa, espera-se que os resultados possibilitem a produção de açôes e estratégias assistenciais de enfermagem seguras voltadas aos pacientes oncológicos em quimioterapia antineoplásica, conforme a realidade vivida pelos sujeitos. Além de servir como subsídio para discussóes sobre o planejamento de intervençóes que proporcionem melhoria da qualidade do atendimento.

A amostra do estudo pode ser considerada um fator limitante, haja vista não ter sido aleatória na fase de observação e apresentar um número amostral reduzido, permitindo considerar somente os resultados aqui encontrados.

\section{CONTRIBUIÇÕES}

Aline Gonçalves da Costa e Aline Maria Pereira Cruz Ramos contribuíram na concepção e planejamento do projeto de pesquisa, na obtenção, análise e interpretação dos dados. Marta Solange Camarinha Ramos Costa e Elisângela da Silva Ferreira contribuíram na revisão crítica. Priscila Cristina de Sousa, Marília Monteiro dos Santos e Danielle Etienne de Oliveira Bezerra Lima contribuíram na revisão crítica e intelectual. Todos aprovaram a versão final para publicação.

\section{DECLARAÇÃO DE CONFLITO DE INTERESSES}

Nada a declarar.

\section{FONTES DE FINANCIAMENTO}

Não há.

\section{REFERÊNCIAS}

1. Ministério da Saúde (BR). Programa Nacional de Segurança do Paciente [Internet]. Brasília: Ministério da Saúde; 2017 [acesso 2017 Ago 23]. Disponível em: http://portalms.saude.gov.br/acoes-e-programas/ programa-nacional-de-seguranca-do-paciente-pnsp/ sobre-o-programa .

2. Ministério da Saúde (BR). Portaria no 529 , de $1^{\circ}$ de abril de 2013 [Internet]. [acesso 2017 Ago 23]. Disponível em: http://www.saude.mt.gov.br/upload/controle-infeccoes/ pasta2/portaria-msgm-n-529-de-01-04-2013.pdf.

3. Urbanetto JS, Gerhardt LM, Guirardello EB, organizador. Segurança do paciente: avanços e desafios para a enfermagem [Internet]. 10 Congresso Internacional da Rede Brasileira de Enfermagem e Segurança do Paciente; 2016 Mar 8-10; Campinas, SP. Campinas,
SP: UNICAMP; 2016. [acesso 2017 Ago 30] 303 p. Disponível em: http://www.rebraensp.com.br/ phocadownload/publicacoes/ANAIS\%20I\%20 CIREBRAENSP\%202016\%20FINAL\%20Ajuste\%20 set16.pdf.

4. Macedo TR, Rocha PK, Tomazoni A, Souza S, Anders JC, Davis K. Cultura de segurança do paciente na perspectiva da equipe de enfermagem de emergências pediátricas. Rev Esc Enferm USP. 2016;50(5):756-762. doi: http:// dx.doi.org/10.1590/s0080-623420160000600007.

5. Livinalli, A. Erros de medicação em oncologia. Rev Onco. 2012;2(10):22-27. [acesso 2017 Ago 30]. Disponível em: http://revistaonco.com.br/wp-content/ uploads/2012/03farmacia.pdf.

6. Rodrigues R, editor. Ordem de infusão de medicamentos antineoplásicos: sistematização de informaçôes para auxiliar a discussão e criação de protocolos assistenciais. São Paulo: Atheneu; 2015.

7. Goldspiel B, Hoffman JM, Griffith NL, Goodin S, DeChristoforo R, Montello CM, et al. ASHP guidelines on preventing medication errors with chemotherapy and biotherapy. Am J Health Syst Pharm. 2015;72(8):e6-e35. doi: https://doi.org/10.2146/sp150001.

8. Neuss MN, Polovich M, McNiff K, Esper P, Gilmore TR, LeFebvre KB, et al. 2013 Updated American Society of Clinical Oncology/Oncology Nursing Society Chemotherapy Administration Safety Standards Including Standards for the Safe Administration and Management of Oral Chemotherapy. J Oncol Pract. 2013 Mar;9(2 Suppl):5s-13s. doi: https://doi.org 10.1200/ JOP.2013.000874.

9. Mendes W, Martins M, Rozenfeld S, Travassos C. The assessment of adverse events in hospitals in Brazil. Int J Qual Health Care. 2009;21(4):279-84. doi: https://doi. org/10.1093/intqhc/mzp022.

10. Bonassa EMA, Gato MIR. Terapêutica oncológica para enfermeiros e farmacêuticos. 4. ed. São Paulo: Atheneu; 2012.

11. Bardin L. Análise de conteúdo. Edições 70. [Internet] São Paulo: Ediçóes 70; 2011 [acessado 2017 Ago 20]. 229 p. Disponível em: http://pt.slideshare.net/alasiasantos/ analise-de-conteudo-laurence-bardin.

12. Conselho Federal de Enfermagem. Resolução COFEN No 0545/2017 [Internet]. Brasília: COFEN; 2017 [acesso 2018 Mai 23]. Disponível em: http://www.cofen. gov.br/resolucao-cofen-no-05452017_52030.html.

13. Matos IB, Toassi RFC, Oliveira MC. Profissóes e ocupaçóes de saúde e o processo de feminização: tendências e implicaçôes. Athenea Digital. 2013;13(2):239-44. doi: https://doi.org/10.5565/rev/athenead/v13n2.1119.

14. Costa EC. Segurança na administração de medicamentos antineoplásicos: conhecimentos e açôes de profissionais de enfermagem [dissertação]. Goiânia: Universidade Federal de Goiás, Faculdade de Enfermagem; 2012. 
15. Correia JN, Albach LSP, Albach CA. Extravasamento de quimioterápicos: conhecimentos da equipe de enfermagem. Ciênc. Saúde. 2011;4(1):22-31. doi: http:// dx.doi.org/10.15448/1983-652X.2011.1.9151.

16. Lima IS, Clementino FS, Miranda FAN, Sousa CSM, Brandão ICA, Brasil SKD. Equipe de enfermagem: conhecimentos acerca do manuseio de drogas antineoplásicas. Rev Enferm. 2011;19(1):40-5.

17. Ribeiro TS, Santos VO. Segurança do paciente em quimioterapia. Rev Bras Cancerol. 2015; 61(2):145-153.

18. Bartlett DJ, Childs DS, Breitkopf CR, Grudem ME, Mitchell JL, Looker SA, et al. Chemotherapy acute infusion reactions: a qualitative report of the perspectives of patients with cancer. Am J Hosp Palliat Care. 2018;35(11):1384-1389. doi: https://doi. org/10.1177/1049909118773995.

19. Wolf ZR. Strategies to reduce patient harm from infusion-Associated Medication Errors: a scoping review. J Infus Nurs. 2018 Jan-Feb; 36(1):58-65. doi: https:// doi.org/10.1097/NAN.0000000000000263. Cited in: PubMed; PMID 29293199.

20. Conselho Federal de Enfermagem. Resolução COFEN No 0569/2018 [Internet]. Brasília: COFEN; 2018 [acesso 2018 Mai 29]. Disponível em: http:// www.cofen.gov.br/wp-content/uploads/2018/02/ Resolu\%C3\%A7\%C3\%A3o-569-2018.pdf.

21. Senna MH, Silva CC, Gelbcke FL, Anders JC, Mesquita MPL. A segurança do trabalhador de enfermagem na administração de quimioterápicos antineoplásicos por via endovenosa. Rev Enferm UERJ. 2014;22(5):649-55. doi: http://dx.doi.org/10.12957/reuerj.2014.15513.

22. Canadian Association of Nurses in Oncology. Standards and competencies for cancer chemotherapy nursing practice [Internet]. Vancouver: Canadian Association of Nurses in Oncology; 2013 [cited 2018 Mai 29]. Available from: https://archive.cancercare.on.ca/common/pages/ UserFile.aspx?fileId=156524.

23. Mohsen MM, Fareed ME. Chemotherapy safety protocol for oncology nurses: it's effect on their protective measures practices. Int J Med Health Sci [Internet]. 2013 [cited 2018 Mai 30];7(9):529-37. Available from: https://waset.org/publications/16606/chemotherapysafety-protocol-for-oncology-nurses-it-s-effect-on-theirprotective-measures-practices.

24. National Institute for Occupational Safety and Health; Centers for Disease Control and Prevention. Personal protective equipment for health care workers who work with hazardous drugs [Internet]. Cincinnati: National Institute for Occupational Safety and Health; 2009 [cited 2018 Mai 30]. Available from: https://www.cdc.gov/ niosh/docs/wp-solutions/2009-106/pdfs/2009-106.pdf.

25. Ministério da Saúde (BR). Protocolos básicos de segurança do paciente [Internet]. Brasília: Ministério da Saúde; 2013 Jul 9. Anexo 01, Protocolo para a prática de higienização das mãos em serviços de saúde; [acessado 2017 Set 25]. Disponível em: http:// portalarquivos.saude.gov.br/images/pdf/2014/julho/03/ PROTOCOLO-HIGIENE-DAS-M--OS.pdf.

26. Crickman R. Chemotherapy safe handling: limiting nursing exposure with a hazardous drug control program. Clin J Oncol Nurs. 2017;21(1):73-78. doi: http://dx.doi. org/10.1188/17.CJON.73-78.

27. Fragilidade do paciente oncológico aumenta incidência de queda em 18\%; conheça barreiras para implementar. Instituto Brasileiro para Segurança do Paciente [Internet]. 2017 Out 6 [acesso 2018 Jan 27]. Disponível em: https:// www.segurancadopaciente.com.br/seguranca-e-gestao/ fragilidade-do-paciente-oncologico-aumenta-incidenciade-queda-em-18-conheca-barreiras-para-implementar/.

28. Rede Brasileira de Enfermagem e Segurança do Paciente. Estratégias para a segurança do paciente: manual para profissionais da saúde [Internet]. Porto Alegre: EDIPUCRS; 2013 [acesso 2017 Ago 31]. Disponível em: http://biblioteca.cofen.gov.br/wpcontent/uploads/2017/10/Estrat\%C3\%A9giaspara-seguran $\% \mathrm{C} 3 \% \mathrm{~A} 7 \mathrm{a}$-do-paciente-manual-paraprofissionais-da-sa\%C3\%BAde.pdf.

29. Ministério da Saúde (BR). Protocolos básicos de segurança do paciente [Internet]. Brasília: Ministério da Saúde; 2013 Maio 3. Anexo 01, Protocolo prevenção de quedas; [acessado 2017 Set 29]. Disponível em: http:/www.saude.mt.gov.br/upload/controle-infeccoes/ pasta12/protocolos_cp_n6_2013_prevencao.pdf

30. Ministério da Saúde (BR). Protocolos básicos de segurança do paciente [Internet]. Brasília: Ministério da Saúde; [2014 Jul 3]. Anexo 02, Protocolo de identificação do paciente; [acesso 2017 Set 27]. Disponível em: http:// portalarquivos.saude.gov.br/images/pdf/2014/julho/03/ Protocolo---Identifica----o-do-Paciente.pdf.

31. Gluyas H. Effective communication and teamwork promotes patient safety. Nurs Stand. 2015 Aug 5;29(49):50-7. doi: http://dx.doi.org/10.7748/ ns.29.49.50.e10042 Cited in: PubMed; PMID 26243123

32. Nogueira JWS, Rodrigues MCS. Comunicação efetiva no trabalho em equipe em saúde: desafio para a segurança do paciente. Cogitare Enferm [Internet]. 2015 [acesso 2018 Fev 01];20(3):636-40 Disponível em: https://revistas. ufpr.br/cogitare/article/view/40016/26245.

33. Agência Nacional de Vigilância Sanitária. Resolução RDC No 220/2004. Brasília: ANVISA; 2014. Disponível em: https://www20.anvisa.gov.br/segurancadopaciente/ index.php/legislacao/item/resolucao-rdc-n-220-de-21de-setembro-de-2004.

34. Ministério da Saúde (BR). Protocolos básicos de segurança do paciente [Internet]. Brasília: Ministério da Saúde; [2014 Jul 3]. Anexo 03, Protocolo de segurança na prescrição, uso e administração de medicamentos; [acesso 
2017 Ago 30]. Disponível em: http://portalarquivos. saude.gov.br/images/pdf/2014/julho/03/ProtocoloMedicamentos.pdf.

36. Conselho Federal de Enfermagem. Guia de recomendaçóes para registro de enfermagem no prontuário do paciente e outros documentos de enfermagem. Brasília: Cofen; 2016.

37. Conselho Regional de Enfermagem. Uso seguro de medicamentos: guia para preparo, administração e monitoramento [Internet]. São Paulo: Coren; 2017 [acesso 2018 Jan 03]. Disponível em: http://portal.coren-sp.gov. $\mathrm{br} /$ sites/default/files/uso-seguro-medicamentos.pdf.

38. Chen HC, Lu ZJ, Lee SH. Nurses' experiences in safe handling of chemotherapeutic agents: the Taiwan case. Cancer Nurs. 2016 Sep-Oct;39(5):E29-38. doi: 10.1097 / NCC.0000000000000314. 